\title{
A Decentralized Approach Based on Unknown Input Observers for Actuator Fault Detection and Isolation of a Class of Interconnected Nonlinear Systems
}

\author{
Amir ABBASI ${ }^{1}$, Javad POSHTAN ${ }^{2 *}$, Ali MOAREFIANPOUR ${ }^{1}$ \\ ${ }^{1}$ Department of Electrical Engineering, Science and Research Branch, \\ Islamic Azad University, Tehran, Iran \\ ${ }^{2}$ Iran University of Science and Technology, \\ Narmak, Tehran, 16846-13114, Iran, \\ jposhtan@iust.ac.ir *(corresponding author)
}

\begin{abstract}
This paper presents a novel decentralized scheme for actuator fault detection and isolation of a class of largescale interconnected nonlinear systems. For each of the interconnected subsystems, a local nonlinear unknown input observer (UIO) is designed without the need to communicate with other agents. The interconnected terms are treated as unknown inputs, hence all subsystems are decoupled completely and the information of other subsystems is not needed for fault detection and isolation. In addition to the interconnections, an exogenous disturbance which contains both system and measurement noise is approximately decoupled. To facilitate the observer design, sufficient condition for existence of the designed observer is formulated in terms of a set of linear matrix inequalities (LMIs) and optimal gain matrices are obtained. A simulation example of an automated highway system demonstrates the effectiveness of the proposed methodology.
\end{abstract}

Keywords: Unknown Input Observer (UIO), Large-Scale System, Decentralized Fault detection and Isolation.

\section{Introduction}

With the advancement of last decades' technology, large-scale systems have been developed in many fields such as telecommunication, industrial processes, power generation, space structures, and transportation. To ensure safe and reliable operations of such systems, the design of fault detection and isolation (FDI) schemes is crucial. Due to the special structure of large-scale interconnected systems such as uncertainty, complexity, interconnection among subsystems, and high dimensionality, non-centralized FDI schemes have been developed for these systems. In these schemes, local diagnosers can be designed using local modes of subsystems; however, choosing a scheme depends on tasks of local diagnosers and the type of information exchange [25].

In decentralized schemes, a local diagnoser can detect and isolate faults only in its underlying subsystem. Nonetheless, communication with other local diagnosers is not always needed.

It should also be considered that the need for exchanging information among local diagnosers may cause an increase in costs and moreover, appropriate mechanisms should be considered such as transmission delays and network access [4]. To diagnose other subsystems, distributed schemes are much more practical [13].

As each fault can influence several subsystems, the interconnections among subsystems is a challenge for FDI of interconnected systems. To decouple subsystems completely, abilities of unknown input observers (UIOs) for dealing with the effect of interdependencies among subsystems has been considered for decentralized state estimation; see for example [8-9,19]. In [6], a bank of decentralized observers was designed so that each observer includes the model of the entire system. A distributed FDI scheme based on the UIOs for networks of interconnected second-order linear time-invariant systems was proposed in [18]. In [22], a distributed FDI for large networked systems with uncertainties based on UIOs was designed such that is resilient to network model uncertainties but cannot relax all limitations on interconnections. FDI of singular delayed LPV systems using UIOs was considered in [7].

As most systems can be described as a class of Lipschitz nonlinear systems [10], this paper focuses on a class of large-scale interconnected systems which satisfy the Lipschitz condition and investigates abilities of UIOs in these systems. In [24], a scheme for decentralized actuator fault diagnosis was proposed based on a sliding mode unknown input observer for an 
automated highway system. Despite having the ability to estimate the fault, the need for the knowledge of the interconnections and fault range, computing numerous constants and coordinate transformation are the drawbacks of aforementioned method. A decentralized actuator fault detection scheme was proposed in [25] where interconnection terms are not assumed unknown and the Lipschitz condition should be satisfied. Moreover, some information of other subsystems is needed. In [26], for an automated highway system a distributed FDI scheme was proposed with the assumption of satisfying the Lipschitz condition and exact knowledge of interconnection terms. For a local diagnoser, If FDI of other subsystems is not needed, choosing a decentralized scheme would be more proper. As a result, without the knowledge of interconnection terms, FDI can be performed. A distributed fault detection method for second-order multi-agent systems was considered in [20] under the assumption that the system has zero mean white noise sequences and faults were treated as unknown inputs using UIOs. In [11], a distributed formation control of networked Euler-Lagrange systems was designed in which the dynamic of each agent was described by Euler-Lagrange equation and fault diagnosis was performed. Fault detection for high-order nonlinear multi-agent systems was proposed in [12], which the unknown nonlinear functions are treated as unknown input. Here interdependencies are considered as unknown inputs and all subsystems are decoupled completely. This makes easier the fault detection and isolation and there is not any limitation on interconnections. However, there may be the noise and disturbance in the system and a special structure of UIO is needed to decouple the disturbance and interconnections simultaneously. In this regard, inspired by the UIO designed in [18] and an LMI approach in [2], a decentralized UIO is designed with the ability of decoupling the interconnections and attenuating the exogenous disturbance. The structure of UIO was defined in [3] is similar to later works (for example [5,14-16]) despite the adding abilities of fault estimation and noise filtration. Compared to [5,14-16], here we design UIO in decentralized form and all variables are obtained using LMI technique without the need to compute or try any constant.

The rest of the paper is organized as follows. Section 2 introduces the problem formulation and some definitions and assumptions are given in this section. Section 3 proposes a design procedure of a decentralized observer and related lemmas and theorem. A new decentralized FDI scheme based on UIOs is presented in Section 4. In Section 5, the simulation results of an automated highway system investigate the performance of the proposed scheme.

\section{Problem Formulation}

Consider a large-scale system composed of $N$ subsystems described by

$$
\begin{aligned}
& \dot{x}_{i}(t)=A_{i} x_{i}+B_{i} u_{i}+\Phi_{i}\left(x_{i}\right)+ \\
& \quad+E_{i} h_{i}(x, u)+G_{i} w_{i} \\
& y_{i}=C_{i} x_{i}+D_{i} w_{i} \quad i=1,2, \ldots, N
\end{aligned}
$$

Where $x_{i} \in R^{n_{i}}, \quad u_{i} \in R^{m_{i}} \quad$ and $\quad y_{i} \in R^{p_{i}}$ are respectively the states, known inputs and outputs of the $i$-th subsystem. The vector $x=\left[\begin{array}{lll}x_{1}^{T} & \ldots & x_{N}^{T}\end{array}\right]^{T} \in R^{n}$ is the state vector of the whole system with $u=\left[\begin{array}{lll}u_{1}^{T} & \ldots & u_{N}^{T}\end{array}\right]^{T} \in R^{m}$, $m=\sum_{i=1}^{N} m_{i}$ and $n=\sum_{i=1}^{N} n_{i}$. Here $h_{i}(x, u) \in R^{q_{i}}$ represents the interconnection of the $i$-th subsystem with other subsystems and $w_{i} \in R^{s_{i}}$ is an exogenous disturbance which contains both system and measurement noise. Matrices $A_{i}, B_{i}, C_{i}, D_{i}, E_{i}$ and $G_{i}$ are real and have suitable dimensions.

The following assumptions are considered in designing the observer:

a. The function $\Phi_{i}\left(x_{i}\right)$ satisfies the Lipschitz condition, i.e.,

$$
\left\|\Phi_{i}\left(x_{i}\right)-\Phi_{i}\left(\hat{x}_{i}\right)\right\| \leq \alpha_{i}\left\|x_{i}-\hat{x}_{i}\right\|
$$

Where $\alpha_{i} \in R$ is a Lipschitz constant and is independent of $x_{i}$.

b. The matrix $C_{i}$ is of full row rank and the matrix $E_{i}$ is of full column rank;

c. $\operatorname{Rank}\left(C_{i} E_{i}\right)=\operatorname{Rank}\left(E_{i}\right)$.

Assumptions (b) and (c) imply that the number of unknown inputs that can be decoupled are at most as many as the outputs. We can result that for an interconnected system if we want to decouple subsystems using UIOs, the number of interactions with other subsystems should be less than or equal to the locally measured outputs. 


\section{Decentralized Observer Design}

We propose the observer for each subsystem with the following form

$$
\begin{aligned}
& \dot{z}_{i}=N_{i} z_{i}+J_{i} u_{i}+L_{i} y_{i}+M_{i} \Phi_{i}\left(\hat{x}_{i}\right) \\
& \hat{x}_{i}=z_{i}-H_{i} y_{i}
\end{aligned}
$$

Where $\hat{x}_{i}$ and $\hat{y}_{i}$ are estimated state and output of the $i$-th subsystem. The matrices $N_{i}$, $J_{i}, M_{i}$ and $L_{i}$ are defined as

$$
\begin{aligned}
& N_{i}=M_{i} A_{i}=K_{i} C_{i} \\
& J_{i}=M_{i} B_{i} \\
& L_{i}=K_{i}\left(I_{i}+C_{i} H_{i}\right)-M_{i} A_{i} H_{i} \\
& M_{i}=I_{n_{i}}+H_{i} C_{i}
\end{aligned}
$$

$H_{i}$ and $K_{i}$ are chosen by the designer. The state estimation error is defined as

$$
e_{i}=x_{i}-\hat{x}_{i}=M_{i} x_{i}-z_{i}-H_{i} D_{i} w_{i}
$$

Hence the error dynamic is written as

$$
\begin{aligned}
\dot{e}_{i} & =N_{i} e_{i}+\left(M_{i} A_{i}-L_{i} C_{i}-N_{i} M_{i}\right) x_{i}+ \\
& +M_{i} E_{i} h_{i}+M_{i}\left(\Phi_{i}\left(x_{i}\right)-\Phi_{i}\left(\hat{x}_{i}\right)\right)+ \\
& +\left(M_{i} B_{i}-J_{i}\right) u_{i}+\left(M_{i} G_{i}-K_{i} D_{i}\right) w_{i}- \\
& -H_{i} D_{i} \dot{w}_{i}
\end{aligned}
$$

In the light of (4), it is easy to derive

$$
\begin{aligned}
& M_{i} A_{i}-L_{i} C_{i}-N_{i} M_{i}=0 \\
& M_{i} B_{i}-J_{i}=0
\end{aligned}
$$

We should have $M_{i} E_{i}=0$ to decouple each subsystem completely and based on (4) it becomes

$$
H_{i} C_{i} E_{i}=-E_{i}
$$

According to the (b) and (c) assumptions, (8) is satisfied and all possible solutions of it are as follows

$$
H_{i}=-E_{i}\left(C_{i} E_{i}\right)^{+}+Y_{i}\left(I_{p_{i}}-\left(C_{i} E_{i}\right)\left(C_{i} E_{i}\right)^{+}\right)
$$

Where $\left(C_{i} E_{i}\right)^{+}$is generalized inverse of $C_{i} E_{i}$, $I_{p_{i}}$ is an identity matrix and $Y_{i}$ is an arbitrary matrix of suitable dimension [18]. For notational briefing $U_{i}=-E_{i}\left(C_{i} E_{i}\right)^{+}$and $V_{i}=\left(I_{p_{i}}-\left(C_{i} E_{i}\right)\left(C_{i} E_{i}\right)^{+}\right)$are defined, hence (9) becomes

$$
H_{i}=U_{i}+Y_{i} V_{i}
$$

Now (6) is converted to:

$$
\begin{aligned}
\dot{e}_{i} & =N_{i} e_{i}+M_{i}\left(\Phi_{i}\left(x_{i}\right)-\Phi_{i}\left(\hat{x}_{i}\right)\right)+ \\
& +\left(M_{i} G_{i}-K_{i} D_{i}\right) w_{i}-H_{i} D_{i} \dot{w}_{i}
\end{aligned}
$$

The following lemmas are useful for proof of the Theorems 1 and 2.
Lemma 1 [23]. The matrices $D, F$ and $S$ are real with appropriate dimensions and $F$ satisfying $F^{T} F \leq 1$. Then for any scalars $\varepsilon>0$ and vectors $x, y \in R^{n}$, we have

$$
2 x^{T} D F S y \leq \varepsilon^{-1} x^{T} D D^{T} x+\varepsilon y^{T} S^{T} S y
$$

Lemma 2 (Schur complement) [1]. Assume $S=\left[\begin{array}{cc}S_{11} & S_{12} \\ * & S_{22}\end{array}\right]$ is a symmetric matrix. $S<0$ is equivalent to $S_{22}<0$ and $S_{11}-S_{12} S_{22}^{-1} S_{12}^{T}<0$.

Theorem 1: Consider the system (1) with the assumptions (a), (b), (c) and $w_{i}=0$, there exists an unknown input observer in the form of (3) if there exists matrices $\bar{K}_{i}, \bar{Y}_{i}$ and $\bar{P}_{i}$ such that

$\left[\begin{array}{cc}X_{i} & X_{i 2} \\ X_{i 2}^{T} & -I_{n_{i}}\end{array}\right]<0$

Where the matrix elements are defined as

$$
\begin{aligned}
X_{i} & =P_{i}\left(I+U_{i} C_{i}\right) A_{i}+\left(\left(I_{i}+U_{i} C_{i}\right) A_{i}\right)^{T} P_{i}+ \\
& +\bar{Y}_{i}\left(V_{i} C_{i} A_{i}\right)+\left(V_{i} C_{i} A_{i}\right)^{T} \bar{Y}_{i}^{T}- \\
& -\bar{K}_{i} C_{i}-C_{i}^{T} \bar{K}_{i}^{T}+\alpha_{i} I_{n_{i}}
\end{aligned}
$$

Proof. Consider the following Lyapunov function of $V_{i}(t)=e_{i}^{T}(t) P_{i} e_{i}(t)$, differentiating this function with respect to time and following from (11) and (2) one has

$$
\begin{aligned}
\dot{V}_{i}=e_{i}^{T} & N_{i}^{T} P_{i}+P_{i} N_{i} e_{i}+ \\
& +2 e_{i}^{T} P_{i} M_{i}\left(\Phi_{i}\left(x_{i}\right)-\Phi_{i}\left(\hat{x}_{i}\right)\right) \leq \\
\leq e_{i}^{T} & N_{i}^{T} P_{i}+P_{i} N_{i} e_{i}+ \\
& \quad+2\left\|e_{i}^{T} P_{i} M_{i}\right\|\left\|\Phi_{i}\left(x_{i}\right)-\Phi_{i}\left(\hat{x}_{i}\right)\right\| \leq \\
\leq & e_{i}^{T} N_{i}^{T} P_{i}+P_{i} N_{i} e_{i}+2\left\|e_{i}^{T} P_{i} M_{i}\right\| \alpha_{i}\left\|e_{i}\right\|
\end{aligned}
$$

We can continue the inequality in (18) using Lemma 1 as follows

$$
\begin{aligned}
\dot{V}_{i} & \leq e_{i}^{T} N_{i}^{T} P_{i}+P_{i} N_{i} e_{i}+\alpha_{i}\left(\left\|e_{i}^{T} P_{i} M_{i}\right\|^{2}+\left\|e_{i}\right\|^{2}\right) \\
& =e_{i}^{T}\left(N_{i}^{T} P_{i}+P_{i} N_{i}+\alpha_{i} P_{i} M_{i} M_{i}^{T} P_{i}+\alpha_{i} I_{n_{i}}\right) e_{i}
\end{aligned}
$$

The following inequality relation should hold to satisfy the Lyapunov stability criteria

$$
N_{i}^{T} P_{i}+P_{i} N_{i}+\alpha_{i} P_{i} M_{i} M_{i}^{T} P_{i}+\alpha_{i} I_{n_{i}}<0
$$

If (20) is satisfied, e(t) tends to zero asymptotically for any initial value of $\mathrm{e}(0)$. To 
convert (20) into a linear matrix inequality, we substitute (4) and (10) in to (20) to get

$$
\begin{aligned}
& P_{i}\left(I_{i}+U_{i} C_{i}\right) A_{i}+\left(\left(I_{i}+U_{i} C_{i}\right) A_{i}\right)^{T} P_{i}+ \\
& +P_{i} Y_{i} V_{i} C_{i} A_{i}+V_{i}^{T} C_{i}^{T} A_{i}^{T} Y_{i}^{T} P_{i}- \\
& -P_{i} K_{i} C_{i}-C_{i}^{T} K_{i}^{T} P_{i}+ \\
& +\alpha_{i}\left(P_{i}\left(I_{i}+U_{i} C_{i}\right)+P Y_{i} V_{i} C_{i}\right) \\
& \left(P_{i}\left(I_{i}+U_{i} C_{i}\right)+P_{i} Y_{i} V_{i} C_{i}\right)^{T}+\alpha_{i} I_{n_{i}}<0
\end{aligned}
$$

For converting to an LMI contains $Y_{i}, K_{i}$ and $P_{i}$, the terms $P_{i} Y_{i}$ and $P_{i} K_{i}$ are nonlinear, so we substitute $\bar{K}_{i}=P_{i} K_{i}$ and $\bar{Y}_{i}=P_{i} Y_{i}$ in (21) to get

$$
\begin{aligned}
& \left(\left(I_{i}+U_{i} C_{i}\right) A_{i}\right)^{T} P_{i}+P_{i}\left(I_{i}+U_{i} C_{i}\right) A_{i}+ \\
& +\left(V_{i} C_{i} A_{i}\right)^{T} \bar{Y}_{i}^{T}+\bar{Y}_{i}\left(V_{i} C_{i} A_{i}\right)-C_{i} \bar{K}_{i}^{T}- \\
& -\bar{K}_{i} C_{i}+\alpha_{i}\left(P_{i}\left(I_{i}+U_{i} C_{i}\right)+\bar{Y}_{i}\left(V_{i} C_{i}\right)\right) \\
& \left(P_{i}\left(I_{i}+U_{i} C_{i}\right)+\bar{Y}_{i}\left(V_{i} C_{i}\right)\right)^{T}+\alpha_{i} I_{n_{i}}<0
\end{aligned}
$$

Based on Lemma 2, (22) is convertible to an LMI by the following form

$$
\left[\begin{array}{cc}
X_{i} & X_{i 2} \\
X_{i 2}^{T} & -I_{n_{i}}
\end{array}\right]<0
$$

where the matrix elements are as Theorem 1 and this completes the proof.

The following theorem gives a sufficient condition for (11) to be stable for $w_{i}=0$ and $\left\|e_{i}\right\|_{2}<\mu_{i}\left\|w_{i}\right\|_{2}$ for $w_{i} \neq 0$.

Theorem 2. Consider the system (1) under assumptions (a), (b), (c) together with the nonlinear observer (3). There exist matrices $\bar{K}_{i}, \bar{Y}_{i}, P_{i}$ and disturbance tuning parameter $\mu_{i}>0$ such that the state estimation error (11) produced by the observer (3) tends to zero asymptotically for $w_{i}=0$ and $\left\|e_{i}\right\|_{2}<\sqrt{ } \mu_{i}\left\|w_{i}\right\|_{2}$ for $w_{i} \neq 0$ if the following LMI optimization problem has a feasible solution:

$$
\begin{aligned}
& \min \left(\mu_{i}\right) \text {, s.t. } \\
& {\left[\begin{array}{cccc}
X_{i} & X_{i 2} & \Omega_{i 1} & \Omega_{i 2} \\
X_{i 2}^{T} & -I_{n_{i}} & 0 & 0 \\
\Omega_{i 1}^{T} & 0 & -\mu_{i} I_{n_{i}} & 0 \\
\Omega_{i 2}^{T} & 0 & 0 & -\mu_{i} I_{n_{i}}
\end{array}\right]<0}
\end{aligned}
$$

where the matrix elements are defined as

$$
\begin{aligned}
X_{i} & =P_{i}\left(I_{n_{i}}+U_{i} C_{i}\right) A_{i}+ \\
& +\left(\left(I_{n_{i}}+U_{i} C_{i}\right) A_{i}\right)^{T} P_{i}+\bar{Y}_{i}\left(V_{i} C_{i} A_{i}\right)+ \\
& +\left(V_{i} C_{i} A_{i}\right)^{T} \bar{Y}_{i}^{T}-\bar{K}_{i} C_{i}-C_{i}^{T} \bar{K}_{i}^{T}+\alpha_{i} I_{n_{i}}+I_{n_{i}}
\end{aligned}
$$

$$
\begin{aligned}
& X_{i 2}=\sqrt{ } \alpha_{i}\left(P_{i}\left(I_{n_{i}}+U_{i} C_{i}\right)+\bar{Y}_{i}\left(V_{i} C_{i}\right)\right) \\
& \bar{K}_{i}=P_{i} K_{i} \\
& \bar{Y}_{i}=P_{i} Y_{i} \\
& \Omega_{i 1}=P_{i} G_{i}+P_{i} U_{i} C_{i} G_{i}+\bar{Y}_{i} V_{i} C_{i} G_{i}-\bar{K}_{i} D_{i} \\
& \Omega_{i 2}=-P_{i} U_{i} D_{i}-\bar{Y}_{i} V_{i} D_{i}
\end{aligned}
$$

Proof. From Theorem 1, we know that the state estimation error is asymptotically stable if $w_{i}=0$. For $w_{i} \neq 0$ consider the following Lyapunov function of $V_{i}(t)=e_{i}^{T}(t) P_{i} e_{i}(t)$, differentiating this function with respect to time and following from (11), (2) and Lemma 1 one has

$$
\begin{aligned}
\dot{V}_{i}= & e_{i}^{T} N_{i}^{T} P_{i}+P_{i} N_{i} e_{i}+ \\
& +2 e_{i}^{T} P_{i} M_{i}\left(\Phi_{i}\left(x_{i}, t\right)-\Phi_{i}\left(\hat{x}_{i}, t\right)\right)+ \\
& +2 e_{i}^{T} P_{i}\left(M_{i} G_{i}-K_{i} D_{i}\right) w_{i}- \\
& -2 e_{i}^{T} P_{i} H_{i} D_{i} \dot{w}_{i} \leq \\
\leq & e_{i}^{T} N_{i}^{T} P_{i}+P_{i} N_{i} e_{i}+ \\
& +2\left\|e_{i}^{T} P_{i} M_{i}\right\|\left\|\Phi_{i}\left(x_{i}, t\right)-\Phi_{i}\left(\hat{x}_{i}, t\right)\right\|+ \\
& +2 e_{i}^{T} P_{i}\left(M_{i} G_{i}-K_{i} D_{i}\right) w_{i}- \\
& -2 e_{i}^{T} P_{i} H_{i} D_{i} \dot{w}_{i} \leq \\
\leq & e_{i}^{T} N_{i}^{T} P_{i}+P_{i} N_{i} e_{i}+ \\
& +2\left\|e_{i}^{T} P_{i} M_{i}\right\| \alpha_{i}\left\|e_{i}\right\|+ \\
& +2 e_{i}^{T} P_{i}\left(M_{i} G_{i}-K_{i} D_{i}\right) w_{i}- \\
& -2 e_{i}^{T} P_{i} H_{i} D_{i} \dot{w}_{i}
\end{aligned}
$$

We can continue the inequality in (30) using Lemma 1 as follows

$$
\begin{aligned}
\dot{V}_{i} \leq & e_{i}^{T} N_{i}^{T} P_{i}+P_{i} N_{i} e_{i}+ \\
& +\alpha_{i}\left(\left\|e_{i}^{T} P_{i} M_{i}\right\|^{2}+\left\|e_{i}\right\|^{2}\right)+ \\
& +2 e_{i}^{T} P_{i}\left(M_{i} G_{i}-K_{i} D_{i}\right) w_{i}- \\
& -2 e_{i}^{T} P_{i} H_{i} D_{i} \dot{w}_{i}= \\
=e_{i}^{T} & \left(N_{i}^{T} P_{i}+P_{i} N_{i}+\alpha_{i} P_{i} M_{i} M_{i}^{T} P_{i}+\alpha_{i} I_{n_{i}}\right) e_{i}+ \\
& +2 e_{i}^{T} P_{i}\left(M_{i} G_{i}-K_{i} D_{i}\right) w_{i}- \\
& -2 e_{i}^{T} P_{i} H_{i} D_{i} \dot{w}_{i}
\end{aligned}
$$

Letting

$$
\begin{aligned}
J_{i} & =\int_{0}^{\infty}\left(e_{i}^{T} e_{i}-\mu_{i} w_{d_{i}}^{T} w_{d_{i}}+\dot{\mathrm{V}}_{i}(e)\right) d t- \\
& -\int_{0}^{\infty} \dot{\mathrm{V}}_{i}(e) d t
\end{aligned}
$$

Where $w_{d_{i}}=\left[\begin{array}{c}w_{i} \\ \dot{w}_{i}\end{array}\right]$ and from (31) we can conclude (33): 


$$
J_{i}=\int_{0}^{\infty}\left[\begin{array}{lll}
e_{i} & w_{i} & \dot{w}_{i}
\end{array}\right] \Psi_{i}\left[\begin{array}{c}
e_{i} \\
w_{i} \\
\dot{w}_{i}
\end{array}\right] d t-\int_{0}^{\infty} \dot{\mathrm{V}}_{i}(e) d t
$$

where

$$
\begin{aligned}
\Psi_{i} & =\left[\begin{array}{ccc}
\Sigma & P_{i}\left(M_{i} G_{i}-K_{i} D_{i}\right) & -P_{i} H_{i} D_{i} \\
* & -\mu_{i} I_{n_{i}} & 0 \\
* & 0 & -\mu_{i} I_{n_{i}}
\end{array}\right] \\
\Sigma & =N_{i}^{T} P_{i}+P_{i} N_{i}+\alpha_{i} P_{i} M_{i} M_{i}^{T} P_{i}+ \\
& +\left(\alpha_{i}+1\right) I_{n_{i}}
\end{aligned}
$$

According to the Lemma 2 and substituting (4) and (10) in to (34), the inequality (24) implies $\Psi_{i}<0$. Under zero initial condition we have $\int_{0}^{\infty} \dot{\mathrm{V}}_{i}(e) d=V_{i}(e)>0$. Therefore, from

one has $J_{i}<0$, indicating $\left\|e_{i}\right\|_{2}<{ }_{i} \mu_{i}\left\|w_{i}\right\|_{2}$. This completes the proof.

\section{Decentralized Fault Detection and Isolation}

While the actuator fault occurs in the $i$-th input, the system described in (1) changes as the following form

$$
\begin{gathered}
\dot{x}_{i}(t)=A_{i} x_{i}+B_{i}\left(u_{i}+f_{i}\left(u_{i}\right)\right)+\Phi_{i}\left(x_{i}\right)+ \\
+E_{i} h_{i}(f(u), x, u)+G_{i} w_{i} \\
y_{i}=C_{i} x_{i}+D_{i} w_{i} \quad i=1,2, \ldots N
\end{gathered}
$$

The unknown function $f_{i}\left(u_{i}(t)\right)$ represents the actuator fault in the $i$-th subsystem. The residual for each subsystem is defined as

$$
r_{i}(t)=y_{i}(t)-\hat{y}_{i}(t)=y_{i}(t)-C \hat{x}_{i}(t)
$$

Since an UIO can estimate the states without considering of the unknown inputs, it is obvious that the residual $r_{i}(t)$ remains within a small boundary, known as threshold value $(T)$, if there is no fault. Otherwise, the residual crosses the threshold value. We can conclude that for each subsystem, if the Euclidean norm of the residual is higher than the threshold value ( $\left.\left\|r_{i}(t)\right\|>T_{i}\right)$, the fault has occurred. The magnitude of the threshold value depends on disturbances, uncertainties, noises and inputs. In addition to the fault, the exogenous disturbance can cause nonzero residual. According to the Theorem 2, as state estimation error satisfies $\left\|e_{i}\right\|_{2}<\bar{u}_{i}\left\|w_{i}\right\|_{2}$, so if $\left\|e_{i}\right\|_{2}>\mu_{i}\left\|w_{i}\right\|_{2}$ we can conclude a fault has occurred. Hence when $w_{i} \neq 0$ a threshold shall be defined such that

$$
T_{i}>\sqrt{ } \mu_{i}\left\|w_{i}\right\|_{2}
$$

Occurrence of the fault changes (11) as the following form

$$
\begin{aligned}
\dot{e}_{i} & =N_{i} e_{i}+M_{i}\left(\Phi_{i}\left(x_{i}\right)-\Phi_{i}\left(\hat{x}_{i}\right)\right)+ \\
& +\left(M_{i} G_{i}-K_{i} D_{i}\right) w_{i}- \\
& -H_{i} D_{i} w_{i}+M_{i} B_{i} f_{i}\left(u_{i}\right)
\end{aligned}
$$

While the fault occurs, if $M_{i} B_{i}$ is nonzero the residual signal will be nonzero. It can be concluded from (4) to satisfy this condition we should have

$$
H_{i} C_{i} B_{i} \neq-B_{i}
$$

All possible solutions of (40) can be written as

$$
H_{i} \neq-B_{i}\left(C_{i} B_{i}\right)^{+}+Y_{i}\left(I_{p_{i}}-\left(C_{i} B_{i}\right)\left(C_{i} B_{i}\right)^{+}\right)
$$

Unfortunately the sufficient condition for satisfying (41) cannot be specified exactly due to lack of specific ways for calculating the value of $Y_{i}$. We can define $Y_{i}$ inside the LMI to get a value that is very proper for the purpose of state estimation; however (41) may not be satisfied. Hence, in this case we can try other methods to define $Y_{i}$ (for example [18]) which lead to the satisfying results.

When the function $h_{i}(f(u), x, u)$ is decoupled completely, the observer of each subsystem is influenced by its own actuator fault. On the other hand, in (36) only the term $f_{i}\left(u_{i}\right)$ has effect on the $i$-th subsystem. As all subsystems are decoupled completely and interconnections have not any effect, each actuator fault affects only its corresponding subsystem and therefore the fault of each subsystem is isolated.

\section{Example}

With the expansion of the traffic problem, automated highway systems have attracted a lot of attention. It can reduce the number of driving accidents by decreasing human errors. In designing such systems, reliability and safety are most important, and therefore designing FDI for the automated highway system is necessary.

According to [21], the longitudinal dynamics of a vehicle-following system to maintain an appropriate forward velocity and safe distance is described by 


$$
\begin{aligned}
& \dot{\Psi}_{i}=v_{i}-v_{(i-1)} \\
& \dot{v}_{i}=\frac{1}{m_{i}}\left(-A_{i \rho} v_{i}^{2}-d_{i}+\zeta_{i}\right) \\
& \dot{\zeta}_{i}=\frac{1}{\tau_{i}}\left(-\zeta_{i}+u_{i}\right) \\
& y_{i}=\left[\begin{array}{c}
\Psi_{i}+\rho_{c} v_{i} \\
\zeta_{i}
\end{array}\right]
\end{aligned}
$$

Where $\Psi_{i}$ is the distance between the $i$-th and the $(i-1)$-th vehicle, $v_{i}$ is the velocity of $i$-th vehicle, $\zeta_{i}$ is the braking/driving force applied to the longitudinal dynamics of the $i$ th vehicle, $m_{i}$ is the mass of the $i$-th vehicle, $A_{i \rho}$ is the aerodynamic drag, $\rho_{c}$ is a positive constant, $d_{i}$ is the constant frictional force and $\tau_{i}$ is the engine/brake time constant. The control input is $u_{i}$ where $u_{i}>0$ describes a throttle input and $u_{i}<0$ describes a brake input and $y_{i}$ is output of the $i$-th vehicle. As the local diagnosers (UIOs) are decoupled completely, the number of vehicles does not matter. Here we assume there are three vehicles and the values are as follows

$$
\begin{aligned}
& m_{i}=1300 \mathrm{~kg}, \\
& A_{i \rho}=0.3 \mathrm{Ns}^{2} / \mathrm{m}^{2}, \\
& d_{i}=100 \mathrm{~N}, \\
& \tau_{i}=0.2 \mathrm{~s}, \\
& \rho_{c}=0.4, \\
& v_{0}=10 \mathrm{~m} / \mathrm{s}
\end{aligned}
$$

The system states are $x_{i 1}=\Psi_{i}, x_{i 2}=v_{i}$ and $x_{i 3}=\xi_{i}$ for $i=1,2,3$. Based on the numerical values of model parameters, the system is described as follows:

$$
\begin{aligned}
\dot{x}_{i} & =\left[\begin{array}{ccc}
0 & 0 & 1 \\
0 & 1 & \frac{1}{1300} \\
0 & 0 & -5
\end{array}\right] x_{i}+\left[\begin{array}{c}
0 \\
-\frac{1}{13}-\frac{0.3}{1300} x_{i 2}^{2} \\
5 u_{i}
\end{array}\right]+ \\
& +\left[\begin{array}{l}
0 \\
0 \\
5
\end{array}\right] f_{i}\left(u_{i}, t\right)-\left[\begin{array}{c}
x_{(i-1) 2} \\
0 \\
0
\end{array}\right] \\
y_{i} & =\left[\begin{array}{lll}
1 & 0.4 & 0 \\
0 & 0 & 1
\end{array}\right] x_{i} \quad i=1,2,3
\end{aligned}
$$

In (44), $x_{(i-1) 2}$ represents the second state (velocity) of previous vehicle. The system matrices in the form of (1) are as follows

$$
\begin{aligned}
& A_{i}=\left[\begin{array}{ccc}
0 & 0 & 1 \\
0 & 1 & \frac{1}{1300} \\
0 & 0 & -5
\end{array}\right], B_{i}=\left[\begin{array}{l}
0 \\
0 \\
5
\end{array}\right] \\
& E_{i}=\left[\begin{array}{c}
\frac{1}{1300} \\
0 \\
0
\end{array}\right], \Phi_{i}=\left[\begin{array}{c}
0 \\
-\frac{1}{13}-\frac{0.3}{1300} x_{i 2}^{2} \\
0
\end{array}\right] \\
& h_{i}=x_{(i-1) 2}
\end{aligned}
$$

The Lipschitz constant according to [10] is $\alpha=0.0069$. Using Matlab LMI toolbox, the minimum value of the disturbance tuning parameter for each subsystem is obtained as $\mu_{i}=0.0282$. The exogenous disturbance is supposed to be $w_{i}=\sin (10 t)$, hence in the light of (38) the fixed threshold value is calculated as $T_{i}=.1679$. In addition, we consider $G_{i}=I_{3 \times 1}$ and $D_{i}=I_{2 \times 1}$. After solving the LMI defined in the Theorem 2, the obtained gain matrices for UIO are as follows

$$
\begin{aligned}
& H_{i}=\left[\begin{array}{cc}
-1 & 1 \\
0 & 0 \\
0 & 0
\end{array}\right] \\
& K_{i}=\left[\begin{array}{cc}
2.74 & -2.14 \\
0.74 & 0.25 \\
2.54 & -1.54
\end{array}\right] \\
& L_{i}=\left[\begin{array}{cc}
0 & 0.6 \\
0 & 1 \\
0 & 1
\end{array}\right]
\end{aligned}
$$

As the fault only has the effect on the third state and the value of this state is available, here we define the residual as $r_{i 3}(t)=x_{i 3}(t)-\hat{x}_{i 3}(t)$. Figure 1 shows the effect of an actuator fault without any disturbance on the first vehicle. In this figure, the third state (braking/driving force) of three vehicles on which the fault can affect it, have been depicted. As expected the fault only influences the first vehicle while for the other vehicles the corresponding residual signals are zero.

Figure 2 shows the effect of an actuator fault on the second vehicle in the presence of the exogenous disturbance. As indicated in this figure, residual signals of first and third vehicles do not show any fault and only a bit of disturbance has been influenced.

Finally, three different actuator faults are applied to the three vehicles, and the results are depicted in Figure 3. As it is observed from this 

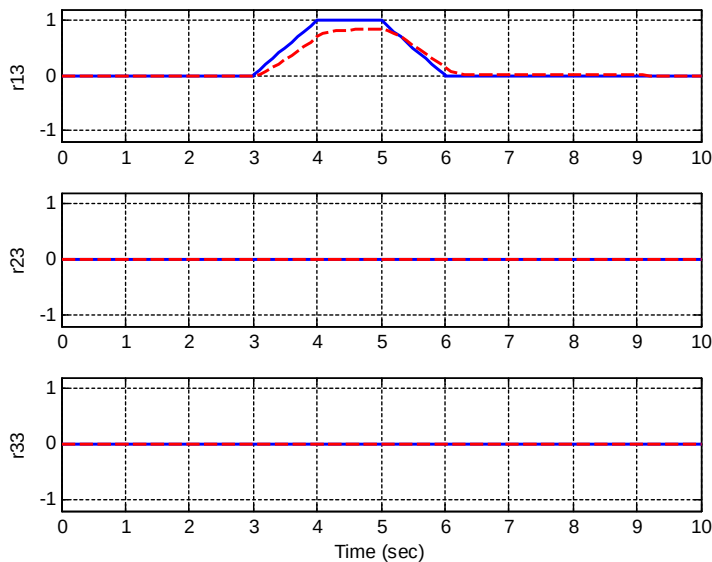

Figure 1. Actuator fault on the first vehicle; solid: fault signal, dashed: residual.
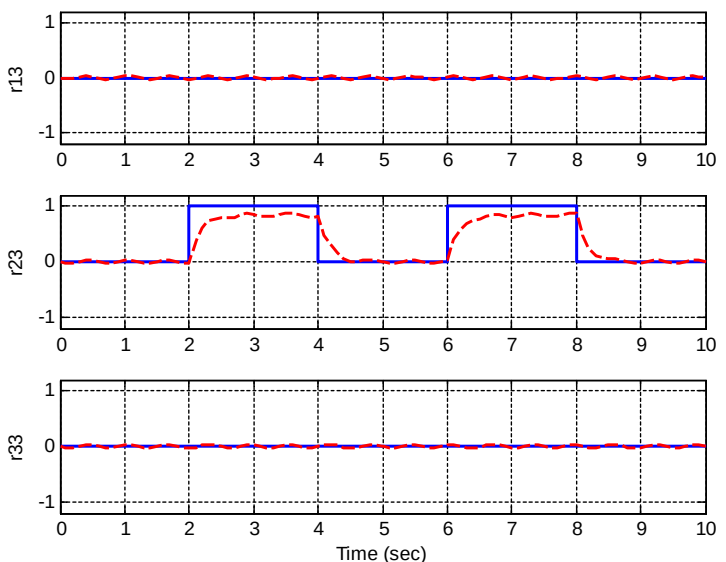

Figure 2. Actuator fault on the second vehicle in the presence of the disturbance; solid: fault signal, dashed: residual.

figure, three residual signals are good representative of fault despite the presence of disturbance.
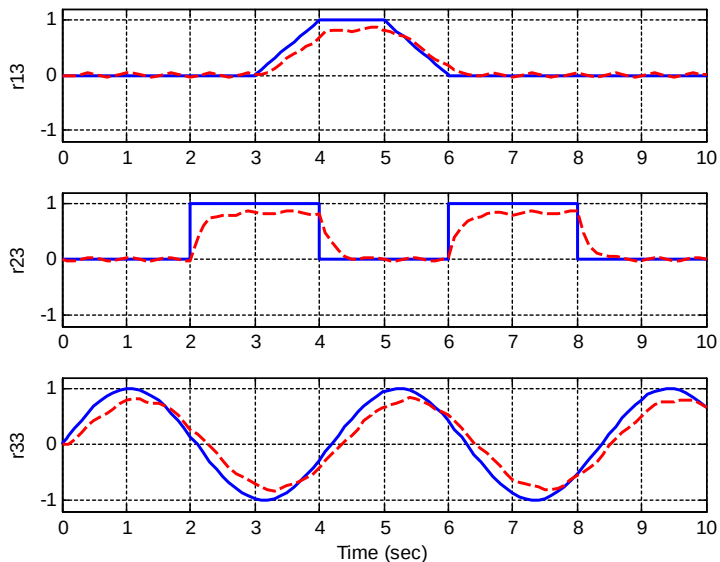

Figure 3. Simultaneous fault on the three cars in the presence of the exogenous disturbance; solid: fault signal, dashed: residual.

\section{Conclusion}

A novel decentralized approach for fault detection and isolation of a class of nonlinear large-scale systems is presented. Capabilities of unknown input observers have been investigated to decouple subsystems for achieving FDI of large-scale interconnected systems. A decentralized unknown input observer using LMI technique has been designed such that both the interconnection and exogenous disturbance can be decoupled. A simulation has been applied to an automated highway system subject to the disturbance shows the simplicity and effectiveness of the proposed methodology for decentralized FDI.

\section{Acknowledgments}

The authors thank Dr Batool Labibi of the Toronto University for her constructive viewpoints.

\section{REFERENCES}

1. BOYD, S. P., L. EL GHAOUI, E. FERON, V. BALAKRISHNAN, V., Linear Matrix Inequalities in System and Control Theory SIAM: Philadelphia,1994.

2. CHEN, W., M. SAIF, Unknown Input Observer Design for a Class of Nonlinear Systems: an LMI Approach, Proc. IEEE Am. Ctrl. Conf., 2006, pp. 834-839.

3. DAROUACH, M., M. ZASADZINSKI, S. J. XU, Full-order Observers for Linear Systems with Unknown Inputs, IEEE Trans. Aut. Ctrl., 39(3), 1994, pp. 606-609.

4. DAVOODI, M. R., K. KHORASANI, H. A. TALEBI, H. R. MOMENI, Distributed Fault Detection and Isolation Filter Design for a Network of Heterogeneous Multiagent Systems, IEEE Trans. Ctrl. Syst. Tech., 22(3), 2014, pp. 1061-1069.

5. DELSHAD, S. S., A. JOHANSSON, M. DAROUACH, T. GUSTAFSSON, Robust State Estimation and Unknown Inputs Reconstruction for a Class of Nonlinear Systems: Multiobjective Approach, Automatica, 64, 2016, pp. 1-7.

6. DING, S. X., P. ZHANG, C. CHIHAIA, W. LI, Y. WANG, E. L. DING, Advanced Design Scheme for Fault Tolerant Distributed 
Networked Control Systems, IFAC Proc. Vol., 41(2), 2008, pp. 13569-13574.

7. HASSANABADI, A. H., M. SHAFIEE, V. PUIG, UIO Design for Singular Delayed LPV Systems with Application to Actuator Fault Detection and Isolation, Intl. J. Syst. Sc., 47(1), 2016, pp. 107-121.

8. HEYDARI, M., M. A. DEMETRIOU, Adaptive Distributed Unknown Input Observers for Interconnected Linear Descriptor Systems, Intl. J. Syst. Sc., 2016, pp. 1-8.

9. HOU, M., P. C. MÜLlER, Design of Decentralized Linear State Function Observers, Automatica, vol. 30(11), 1994, pp. 1801-1805.

10. KHALIL, H. K., J. W. GRIZZLE, Nonlinear Systems (Vol. 3), New Jersey: Prentice hall, 1996.

11. LIU, L., J. SHAN, Distributed Formation Control of Networked Euler-Lagrange Systems with Fault Diagnosis, J. Franklin Inst., 352(3), 2015, pp. 952-973.

12. LIU, X., X. GAO, J. HAN, Observerbased Fault Detection for High-order Nonlinear Multi-agent Systems, J. Franklin Inst., 353(1), 2016, pp. 72-94.

13. LIU, X., X. GAO, J. HAN, Robust Unknown Input Observer based Fault Detection for High-order Multi-agent Systems with Disturbances, ISA Trans., 61, 2016, pp. 15-28.

14. LIU, X., Z. GAO, Unknown Input Observers for Fault Diagnosis in Lipschitz Nonlinear Systems, IEEE International Conference on Mechatronics and Automation, 2015, pp. 1555-1560.

15. MOHAMED, K., M. CHADLI, M. CHAABANE, Unknown Inputs Observer for a Class of Nonlinear Uncertain Systems: An LMI Approach, Intl. J. Aut. and Comp., 9(3), 2012, pp. 331-336.

16. MONDAL, S., G. CHAKRABORTY, K. BHATTACHARYY, LMI Approach to Robust Unknown Input Observer Design for Continuous Systems with Noise and Uncertainties, Intl. J. Ctrl, Aut. and Syst., 8(2), 2010, pp. 210-219.

17. REPPA, V., M. M. POLYCARPOU, C. G. PANAYIOTOU, Decentralized Isolation of
Multiple Sensor Faults in Large-scale Interconnected Nonlinear Systems, IEEE Trans. Aut. Ctrl, 60(6), 2015, pp. 1582-1596.

18. SHAMES, I., TEIXEIRA, A.M., SANDBERG, H., JOHANSSON, K.H., Distributed Fault Detection for Interconnected Second-order Systems, Automatica, 47(12), 2011, pp. 2757-2764.

19. SHI, J., X. HE, Z. WANG, D. ZHOU, Distributed Fault Detection for a Class of Second-order Multi-agent Systems: An Optimal Robust Observer Approach. IET Control Theory \& Apps., 8(12), 2014, pp. 1032-1044.

20. SHI, J., X. HE, Z. WANG, D. ZHOU, Distributed Fault Detection for a Class of Second-order Multi-agent Systems: An Optimal Robust Observer Approach, IET Control Theory \& Applications, 2013, 8(12), pp. 1032-1044.

21. SPOONER, J. T., K. M. PASSINO, Faulttolerant Control for Automated Highway Systems, IEEE Trans. Veh. Tech. 46(3), 1997, pp. 770-785.

22. TEIXEIRA, A., I. SHAMES, H. SANDBERG, K. H. JOHANSSON, Distributed Fault Detection and Isolation Resilient to Network Model Uncertainties, IEEE Trans. Cyber., 44(11), 2014, pp. 2024-2037.

23. WANG, Y., L. XIE, C. E. De SOUZA, Robust Control of a Class of Uncertain Nonlinear Systems, Syst. \& Ctrl. Let., 19(2), 1992, pp. 139-149.

24. YAN, X. G., C. EDWARDS, Robust Decentralized Actuator Fault Detection and Estimation for Large-scale Systems using a Sliding Mode Observer, Intl. J. Ctrl., 81(4), 2008, pp. 591-606.

25. ZHANG, X., Decentralized Fault Detection for a Class of Large-scale Nonlinear Uncertain Systems, Proc. IEEE Am. Ctrl. Conf., 2010, pp. 5650-5655.

26. ZHANG, X., Q. ZHANG, Distributed Fault Detection and Isolation in a Class of Large-scale Nonlinear Uncertain Systems, IFAC Proc. Vols., 44(1), 2011, pp. 4302-4307. 